ARID International Journal for Science and

Technology (AlJST)

ISSN: 2662-009X

Journal home page: http://arid.my/j/aijst

$$
\begin{aligned}
& \text { مَجلةُ أُريد الدَّوليةُ للعُلومِ والتّكنولوجيا } \\
& \text { العدد } 8 \text { ، البجلد } 4 \text { ، كانون الأول } 2021 \text { م }
\end{aligned}
$$

\title{
The Educational Aspects of Electronic Components and Their Related Circuits
}

Hani J. Kbashi ${ }^{1}$ and Salwan K.J. Al-Ani ${ }^{2 *}$

${ }^{12}$ Department of Physics, College of Science, University of Baghdad, Baghdad, Iraq.

${ }^{2}$ Department of Physics, College of Science, Al-Mustansirya University, Baghdad, Iraq.

$$
\begin{aligned}
& \text { الجوانب التعليمية للمكونات الإكترونية والدوائر المرتبطة بها } \\
& \text { هاني جاسم كباثي } 1 \text { و سلو ان كمال جميل العاني2 } \\
& \text { 1. قسم الفيزياء، كلية العلوم، جامعة بغداد، بغداد، العراق } \\
& \text { 2. قسم الفيزياء، كلية العلوم، الجامعة المستتصرية، بغداد، العراق }
\end{aligned}
$$




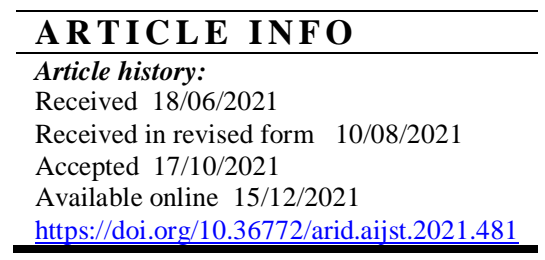

\begin{abstract}
In the modern age, it's almost impossible to imagine a world without electronic items and devices. We take it for granted at the forefront of practical technology, improving daily-based used devices and systems from individual electronic components to the smallest circuit boards for new smartphone systems, with innovation to meet society's communication, technology and energy needs. Therefore, thousands of students, each year, are required to gain in-depth knowledge and experience of basic and high skills of electronic equipment and devices. But the fear and apprehension of the use of electronic pieces, particularly during the online teaching with the difficulties of accessing the laboratories due to the COVID-19 situation, maybe one of the problems of students as they feel that the use of these pieces is difficult and needs external expertise to do them. Nevertheless, nowadays teaching electronics and providing informative sources to a wide spectrum of students from high school, college, and university levels at different departments became an important need for capacity building and training programs. In this article, an informative review of the electronics components and devices supported with simulation results using the Proteus design suite is provided that is useful for both high-level secondary school and the wide level of undergraduate and postgraduate university students.

The research paper has referred, as much as possible, to the hierarchy and the cognitive sequence of using these electronic components such as resistors, capacitors, diode, transistors etc. It is also referred to some electronic circuits and devices such as electronic generator, switching, amplifier that are designed and implemented using the Proteus software which taking into account the learning outcomes and most important physical parameters that can be obtained from each electronic circuit. Then a microprocessors circuits and Arduino have been provided as a compact electronic board for wide range of electronic applications.
\end{abstract}

Keywords: Electronics components, transistors, resistors, diode, integrated circuit, microprocessors circuits, Proteus and Arduino. 


\section{(الملخص:}

في العصر الحديث، يكاد يكون من المستحيل تخيل عالم بدون عناصر وأجزاة إلكترونية، نحن نعتبر ذلك أمراً مسلماً به في في الإني طليعة التكنولوجيا العملية، لتحسين وتطوير الأجهزة والأنظمة الإلكترونية المستخدمة في حياتنا اليومية من المئية المكونات

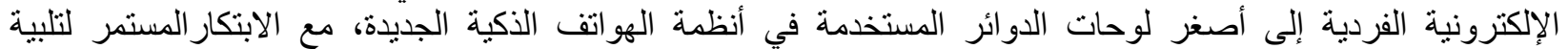

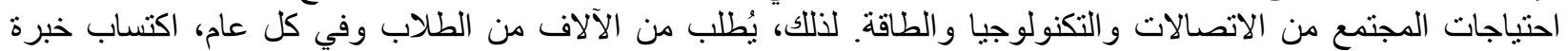

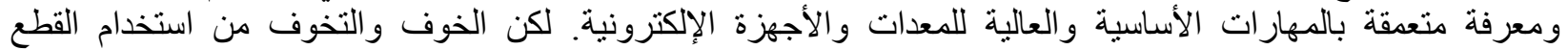

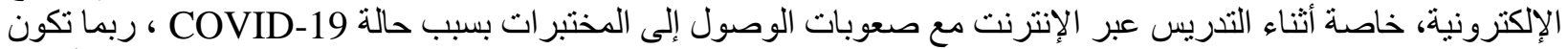

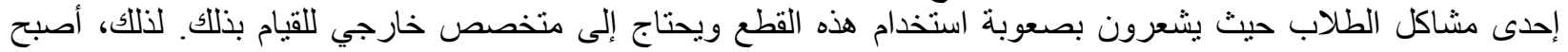

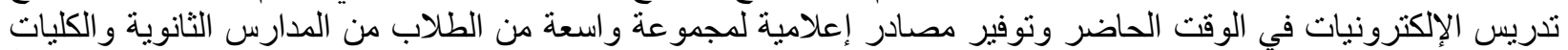

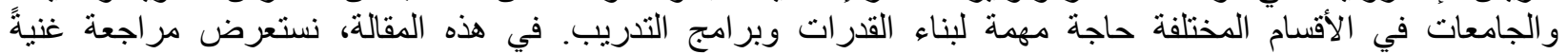

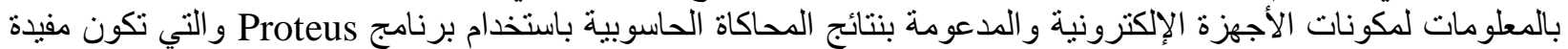

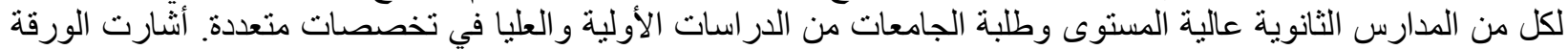

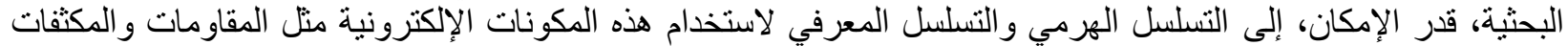

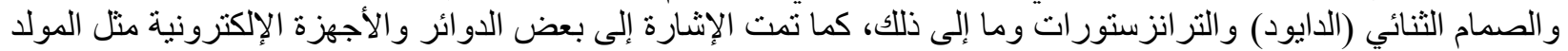

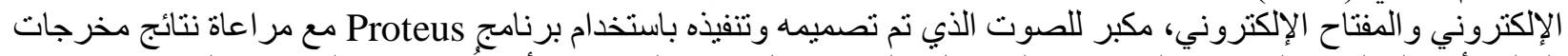

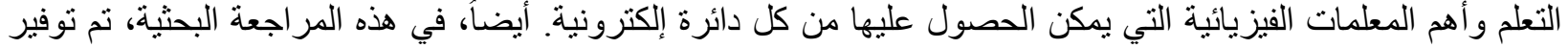
معلومات عن دوائر المعالجات الدقيقة و Arduino كلوحة إلكترونية مدمجة لمجمو عة واسعة من التطبية التبيقات الإلكترونية. 


\section{Introduction}

\section{Paper Structure and Learning Outcomes:}

This paper provides a review and provision information of key electronic components and devices with validation of the electronic circuits using Proteus simulation, hence, provide a compact online solution for electronics laboratories at the engineering and science undergraduate students, particularly, during the COVID-19 pandemic time and lockdown. Proteus simulation is a good opportunity to encourage students to build a virtual electronic circuit and run it using the proteus simulation, therefore, helping them to study their practical module as well as build new electronic circuits and systems. This step will help students with self-learning and feel like a part of a learning community and also present an overview of the key features of the electronic fundamentals that students can refer back to. However, the paper is structured as shown in the chart below.

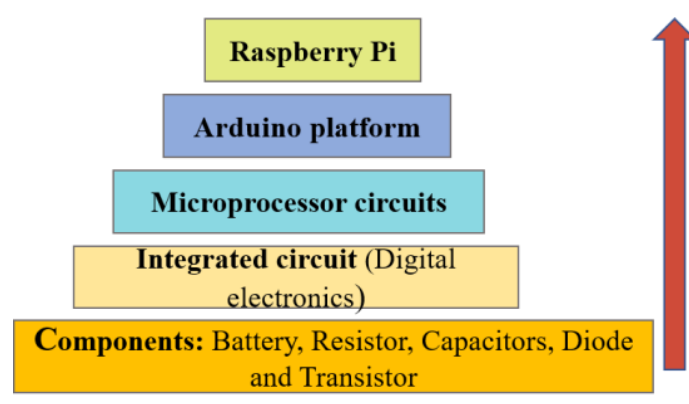

The structure includes the importance of the electronics components with their connection such as the serial and parallel resistors and capacitors connection that are delivered to school students in the high-level to build their knowledge. It also includes the transistors, analog, gates, and digital electronics that have been taught to different academic levels at the university since the $70^{\text {th }}$ of the last century to train students in the designing and implementation of different electronic devices such as an audio amplifier, switching, and so on so far. The Proteus design 
software tool has been used in this paper to simulate some circuits and devices, hence presenting the output results in the form of figures and curves. Moreover, this review also contains the recent new built-in devices such as Arduino and Raspberry pi that have been implemented and become more popular than anticipated for student projects and postgraduate research as well as for young and community hobbies.

Therefore, the learning outcomes of this paper are to be able to:

- Explain how electronic components work such as a resistor, diode, capacitor, and transistor.

- Demonstrate and understand the fundamentals of analogy and digital circuits.

- Demonstrate and understand the Microprocessors and Arduino circuits.

- Help a wide spectrum of students about creating, designing, and characterize circuits, devices, and projects.

- Understand the online teaching with the difficulties of accessing the laboratories due to the COVID-19 situation.

\section{Electronic Components:}

I. Electrical Battery is an electronic component that contains single or many electrochemical cells and external wire connections [1] which converts the stored chemical energy into electrical potential energy through a transient chemical process, after which their activity ceases. This energy is accessible through two terminals, called poles, electrodes, or terminals. One of them is a positive pole which is named the cathode and the other is a negative pole which named anode as shown in the common schematic symbols in Fig. 1 [2]. These two terminals are connected to two different types of metallic plates, called electrodes, that are submerged in chemical materials inside the battery. 


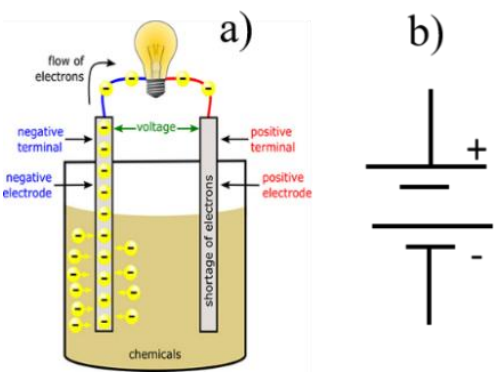

Fig. 1: (a) battery chemical cell and (b) battery schematic symbols.

The chemical liquid interacts with the metals, causing electrons to overflow to build upon the negative electrode (terminal) and producing a lack of electrons on the positive electrode (terminal). Therefore, the negative terminal is the source of electrons flow through an external load circuit such as a lamp to the positive terminal allowing the current to flow from the positive to the negative. In the case of connecting to the external load, the redox interaction will convert high-energy reactants to lower-energy products, hence, the free-energy difference will transfer to the external load circuit in the form of electrical energy [3].

II. Resistor (R): is two terminals passive electrical component that represents the electrical resistance as a circuit member. It is used in the electronic and electrical circuits to reduce current flow from the positive to the negative and hence adjust the flow levels of the electrons. It is also used as a voltage divider, bias active elements, gain controller, and terminate transmission lines, among other uses. It has two common schematic symbols (Fig. 2a) when using it in the circuit diagram. Resistors can have a fixed value which only changes slightly with high temperature, long time, or high operating voltage; It can also have a variable value that can be used to adjust current flow in the circuit such as in temperature (heat) sensing devices, position lighting, humidity sensing, tunable amplifier or chemical activity. The electrical resistance unit is the Ohm as in the symbol of $\Omega$. The ideal resistor behaved according to the relationship specified 
by Ohm's law which states that "the current through a conductor between two points is directly proportional to the voltage across the two points" as follow $[4,5]$ :

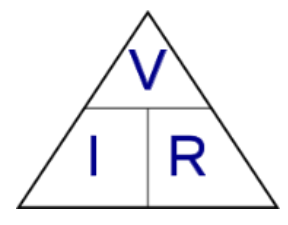

$$
\boldsymbol{V}=\boldsymbol{I} \cdot \boldsymbol{R} \text { Ohm's law triangle }
$$

Where $\mathrm{V}$ is the applied voltage, $\mathrm{I}$ is current flow in the circuit, and $\mathrm{R}$ is the resistance. The equation interchangeability can be illustrated by a triangle shape above.

Resistors can be connected in series and parallel in the electronic circuit $[6,7]$. In seriesconnected as shown in Fig. 2b, the total resistance is the sum of their resistance values, the current is the same across each resistor and voltage is the adding up across each resistor. While in the parallel connection as shown in Fig. 2c, the total resistance is the reciprocal of the sum of the reciprocals of the individual resistors, the current adds up and voltage is the same across each resistor. Table 1 illustrates the features of both series and parallel resistors connection.

The Power dissipation by the resistor is calculated as $P=I V$, hence can be derivative from Ohm's law to become $P=I^{2} R$ or $P=V^{2} / R$, where $V$ is the voltage across the resistor and measured by Volts unit, $I$ is the current passing through the same resistor and measured by amper. This kind of power will convert to thermal energy (heat) which needs to dissipate by the resistor's package or heat sink before the temperature rises dramatically. 
a)

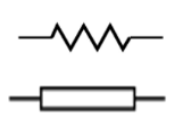

b)

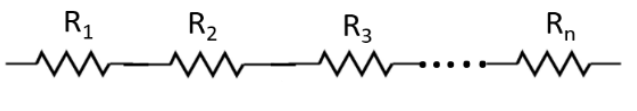

c)

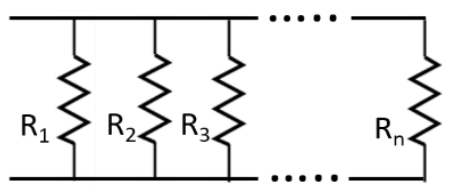

Fig. 2: a) Resistor schematic symbols; b) series connection of the resistors; and c) parallel connection of the resistors.

Table(1): Resistor series and parallel connection features.

\begin{tabular}{|c|c|}
\hline Resistor series connection features & Resistor parallel connection features \\
\hline$R_{T}=R_{1}+R_{2}+R_{3}+-----+R_{n}$ & $\frac{1}{R_{T}}=\frac{1}{R_{1}}+\frac{1}{R_{2}}+\frac{1}{R_{3}}+----+\frac{1}{R_{n}}$ \\
\hline$I_{T}=I_{1}=I_{2}=I_{3}=-----=I_{n}$ & $I_{T}=I_{1}+I_{2}+I_{3}+-----+I_{n}$ \\
\hline$V_{T}=V_{1}+V_{2}+V_{3}+-----+V_{n}$ & $V_{T}=V_{1}=V_{2}=V_{3}+-----+V_{n}$ \\
\hline 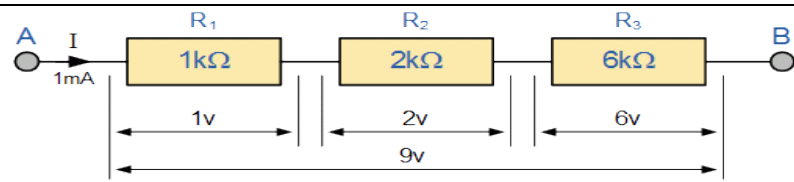 & $R_{1}^{R_{2}}$ \\
\hline
\end{tabular}

In electronics, current-voltage which is called I-V characteristic curve is one of the main graphical curves that could be utilized to define and describe the process and behavior of any electronic component (resistors, diode), circuit (amplifiers), and devices (semiconductors, lasers diodes and solar cells). So, the I-V curve can help students to understand the behavior of the current and voltage combinations in the electronic circuit. Students can also learn from the I-V curve about determining the circuit operation.

For instance, the I-V characteristics of the electronic circuit [8] in Fig 3 a) illustrate the link between the current passing through the resistor and the voltage across the same resistor. So, with the implementation of Ohm's electronic circuit and simulate it using Proteus 8 software, I-V characteristic curves can be generated as shown in Fig. 3b) and students can learn the followings: 
- The current flow through the resistor is a function of the voltage across the same resistor and it is clearly can demonstrate this visually in the linear characteristics curve on both I (forward bias) and III (backward bias) regions.

- In the other words, based on Ohm's law, the flowing current through the resistor is increasing by increase the voltage across the resistor.

- Students can construct from the graph the linear relationship between the voltage and the current (fitting line or slope; V/I) which represents the value of $R$ while $1 / R$ is the relationship between the current and the voltage (inverse of the slope; I/V).

a)

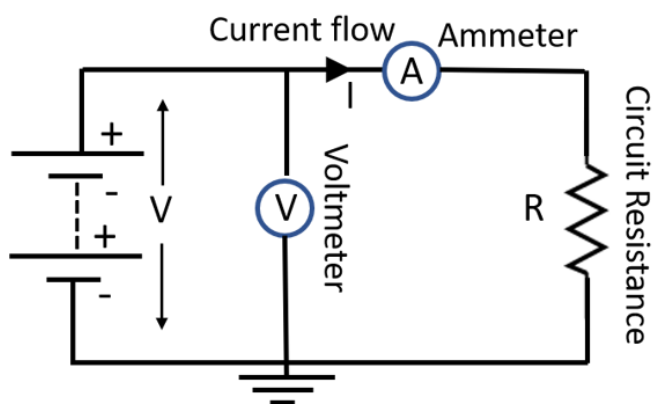

b)

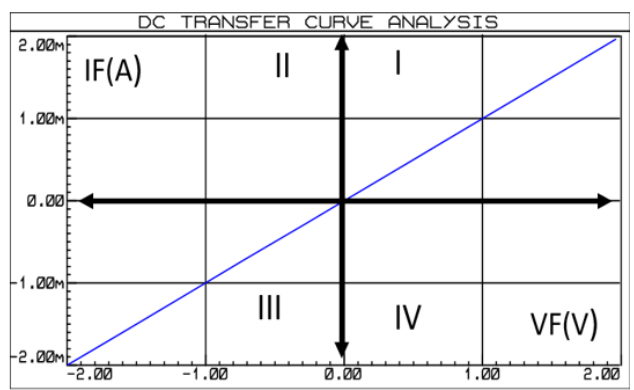

Fig. 3: a) Simple Ohm's electronic circuit [8] and b) its I-V characteristic using Proteus 8 software.

III. Capacitors (C): is a passive component with two distinguished terminals (plats), one is positive and another is negative, The main capacitors task is to store the electrical energy and supplied to the circuit in the form of the electric field. It can also have a fixed and variable value as shown in the common symbol in Fig. 4a. In contrast to the resistance, an ideal capacitor does not suffer from energy dissipation, while in reality, actual capacitors suffer from a very low energy dissipation. When the voltage from the battery is applied across the capacitor two terminals, charges flow onto and from the plates and create an electrical potential difference between the plates. Hence, an electric field develops through electron charge separation where a 
net positive charge accumulates around one plate and the net negative charge accumulates around the second plate. An ideal capacitor is characterized by a constant capacitance (C) that also measures the amount of electrical energy stored (or separated) for a given electric potential. $\mathrm{C}$ which is measured in Farads defined as "the ratio of the positive or negative charge $\mathrm{Q}$ on each conductor to the applied voltage $V^{\prime}$ as given below [9]:

$$
C=Q / V
$$

It can be described as the ability of a body to hold an electric charge. Unlike the resistors, using the schematic diagram in Fig. 4b, capacitors connected in series reveal that the total series is smaller than any of its capacitors in the circuit because it is the reciprocal of the sum of the reciprocals of the individual capacitors. The total capacitor charge is the same as every charge in the circuit. So, the total voltage difference from end to end is the sum of the voltage for every single capacitor. Whereas, the total capacitors connected in parallel as shown in the schematic diagram (Fig. 4c) is the sum of their capacitances values which mean that each capacitor contributes to the total capacitor value, while the charge is distributed among them by size and each capacitor has the same applied voltage. Table 2 illustrate the features of both series and parallel resistors connection

The energy that the capacitor can store is [10] $\frac{1}{2} Q V=\frac{1}{2} C V^{2}$
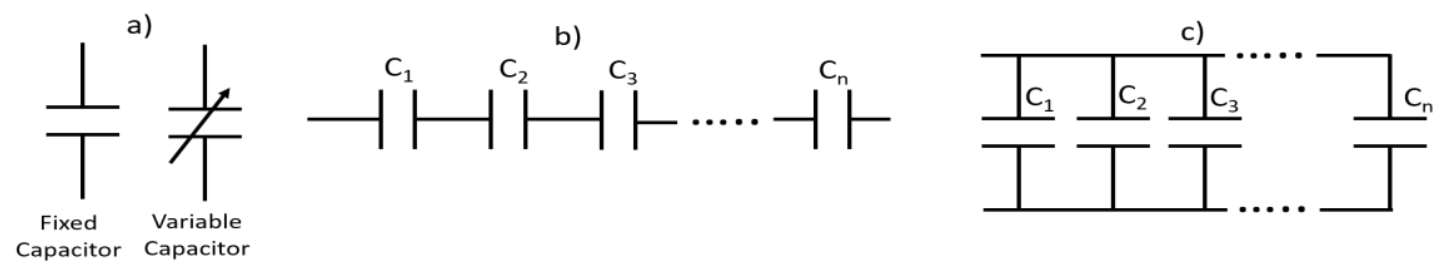

Fig. 4: a) Fixed and variable capacitors schematic symbols; b) capacitors series connection; and c) capacitors parallel connection. 
Table (2): Capacitors series and parallel connection features.

\begin{tabular}{|c|c|}
\hline Capacitors series connection features & Capacitors parallel connection features \\
\hline$\frac{1}{C_{T}}=\frac{1}{C_{1}}+\frac{1}{C_{2}}+\frac{1}{C_{3}}+----+\frac{1}{C_{n}}$ & $C_{T}=C_{1}+C_{2}+C_{3}+-----+C_{n}$ \\
\hline $\begin{array}{c}I_{C T}=I_{C 1}=I_{C 2}=I_{C 3}=----- \\
=I_{C n} \\
Q_{C T}=Q_{C 1}=Q_{C 2}=Q_{C 3}=---- \\
=Q_{C n}\end{array}$ & $\begin{array}{c}I_{C T}=I_{C 1}+I_{C 2}+I_{C 3}=---+I_{C n} \\
Q_{C T}=Q_{C 1}+Q_{C 2}+Q_{C 3}+-----+Q_{C}\end{array}$ \\
\hline$V_{C T}=V_{C 1}+V_{C 2}+V_{C 3}+-----+V_{C r}$ & $V_{C T}=V_{C 1}=V_{C 2}=V_{C 3}+-----+V_{C 1}$ \\
\hline 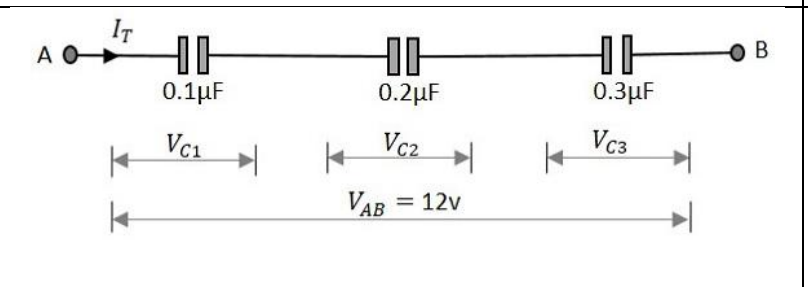 & 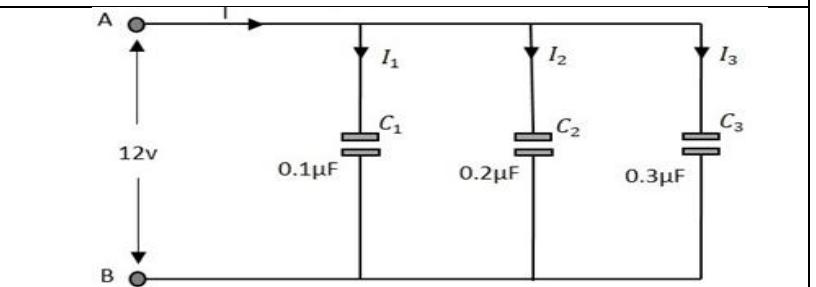 \\
\hline
\end{tabular}

IV. Diode (D): is an active electronic component with two asymmetric conductance terminals. Idea diode has low resistance in one terminal that allows the current to pass forward, and high resistance in the other terminal that prevents the current from passing backward. A crystalline semiconductor diode [11] is the most commonly used type today with a positive (p)-negative (n) junction as shown in Fig. 5a, where the empty circles on the left-side represent the holes and the filled circles on the right side represent the electrons. The depletion layer (region) causes by holes that recombined with free electrons (n-type, negative signs) and electrons that recombined with free holes (p-type, positive signs). Such recombination originates a p-type layer to be negatively charged and the n-type layer to be positively charged forming the depletion region with a potential difference of about $0.7 \mathrm{~V}$ (separation between the p-type and ntype materials). Due to this potential difference, a very small current will flow, and for this reason, an external voltage supply such as a battery that should have a higher potential difference 
can be used to increase the current flow. However, the diode diagram is shown in Fig. $5 \mathrm{~b}$ clarifies the p-n diode symbol with two terminals' names anode which is the positive, and cathode which is negative. However, when the circuit is forward-biased by the applied voltage, the current will flow from p-type towards n-type in the direction of the orange arrow. The diode can also be designed to emit light and called a light-emitting diode (LED) as in Fig. 5c. The main difference between the diode and LED is that the diode allows the current to pass through only one direction and prevents it in the opposite direction while the LED works based upon the electroluminance phenomenon where the semiconductor material emits light when electrons and holes integrated under the influence of the applied voltage as in the LED in the TV remote control and laser pointer.

a)

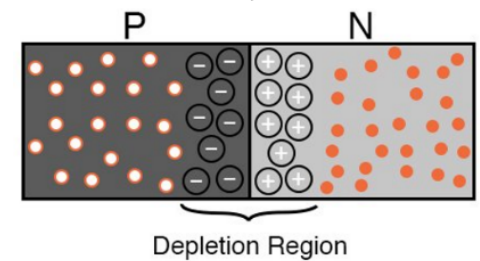

Fig. 5: a) P-N diode junction, b) diode schematic symbols; c) Light-emitting diode schematic symbols.

When the diode is forward biased in the circuit such as in Fig. 6 a), by means of anode positive to the cathode, a forward current passes through the diode and operates in the top right quadrant of its I-V characteristics curves shown in Fig. 6 b) [8]. By simulating the circuit in fig. 6 a) using Proteus 8 software simulation, we plot the I-V characteristic and students can learn from this curve the followings:

- Compared to the I-V linear curve for resistor, diode I-V characteristic curve is nonlinear behavior on both I (forward bias) and III (backward bias) regions. 
- In case of the diode is forward biased, the forward current will not change from zero till the applied voltage exceeds the $0.7 \mathrm{~V}$ of the internal barrier, after that the current will increases dramatically with a distinctly small increase in the applied voltage forming a straight-line upward with fitting line intersect the $\mathrm{x}$-axis at $0.7 \mathrm{~V}$ (the "knee" point on the right side of Fig. 6b)[11].

- On the other side, in the case of the diode is backword (reversed) biased, the diode blocks the current $(I=0)$ until the applied voltage exceeds the breakdown voltage which is $-0.7 \mathrm{~V}$, the reverse current also increases rapidly forming straight-line downward in the lower left quadrant (shaded as a grey).

- $\quad$ This reverse breakdown voltage point is utilized for good effect with Zener diodes [11].

a)

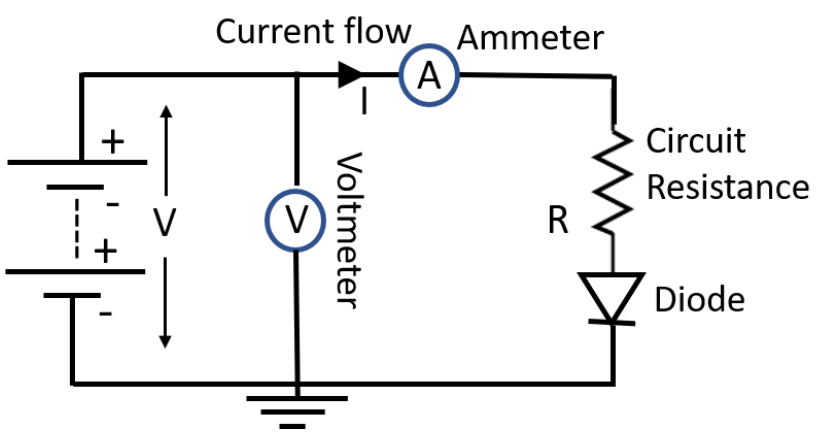

b)

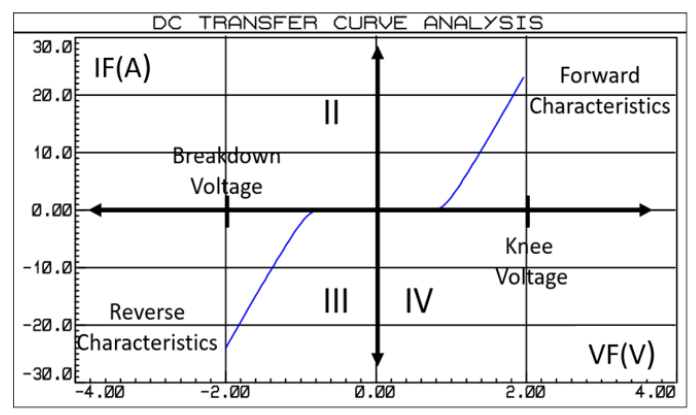

Fig. 6: a) Simple diode-based electronic circuit [8] and b) its I-V characteristic using Proteus 8 software.

V. Transistor $\left(\mathbf{T}_{\mathbf{r}}\right)$ : is a semiconductor active device with three terminals named Base, Collector, and Emitter to connect with the external circuit. The $\operatorname{Tr}$ can be placed in the electronic circuit depends on the application, it works as a switch, amplifier, and power controller. It is composed of semiconductor material ( silicon, germanium, and some other semiconductor materials ) usually with at least three terminals (Base, Collector, and Emitter) for connection to an external circuit. Transistors can be NPN as shown symbol in Fig. 7a or PNP in 
Fig. 7b. For instance, Tr works as a switch by controlling the output voltage or current between the collector and emitter terminals via control the small voltage or current at the base terminal (that is, flowing between the Base and the Emitter) [12]. Likewise, due to the controlled output voltage or current is higher than the input voltage or current, hence produce a stronger output signal. The Tr works here as a signal amplifier and this property is called again which is proportional between the high output signal to the weak input signal as shown in Fig. 7c [13]. For example, in this circuit, a microphone transferred the vocal sounds into an electrical signal which can be amplified using a transistor in some useful way and hence feedback to a loudspeaker, which would replicate the tones originally picked up by the microphone. From this circuit, students can learn:

- How to build a car and mobile audio stereo amplifier.

- They also can learn how to control the gain value which is the division of resistor value

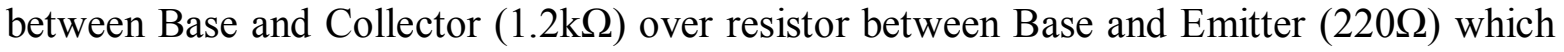
gives a gain of 5.45 .

Alternatively, the transistor can be used as an electrically controlled switch in the circuit to turn the current on or off where the amount of current is determined by other circuit elements. For a field-effect transistor (FET) in Fig. 7d, which is a type of transistor that uses the electric field to control the current flow, has three terminals which are labelled gate, source, and drain. likewise NPN and PNP terminology, FETs can be also N-channel and P channel to control the current flow by the application of a voltage to the gate, which in turn alters the conductivity between the drain and source. However, transistors have developed electronics generations and revolutionized smaller and cheaper devices such as computers, radios and calculators, among 
other things. Today, many $\operatorname{Tr}$ can found instilled in integrated circuits or could also be packaged individually.

a)

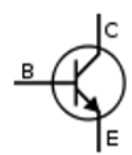

b)

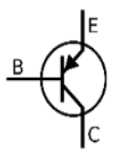

c)

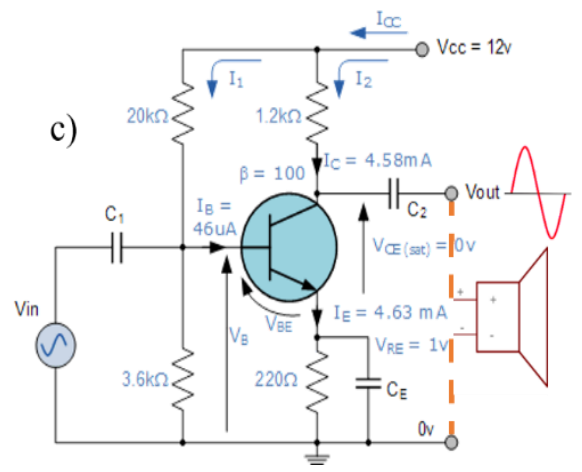

d)

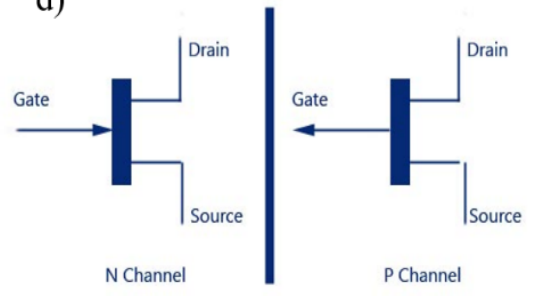

Fig. 7: a) NPN transistor schematic symbols (arrow towards outside); b) PNP transistor schematic symbols (arrow towards inside); c) audio amplifier using the transistor [13] and d) N and P-channel FET.

Figure 8 shows the simulation results using Proteus 8 software for output characteristics for common PNP and NPN configuration.

a)

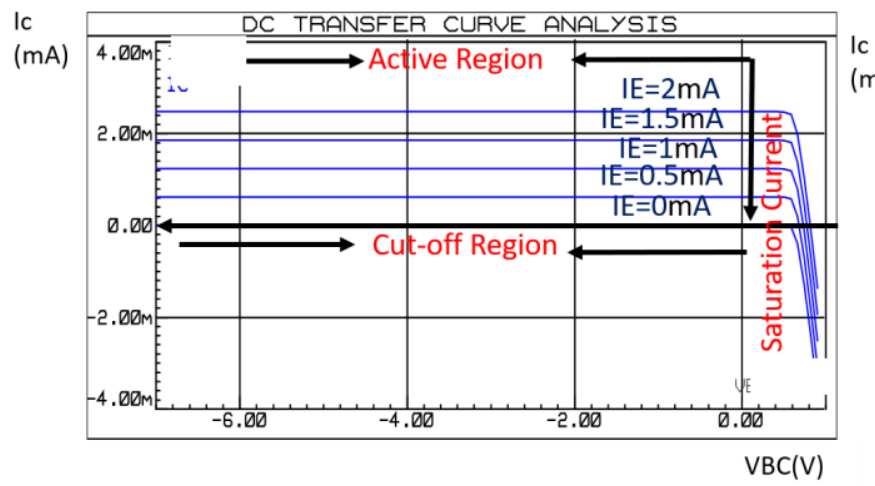

b)

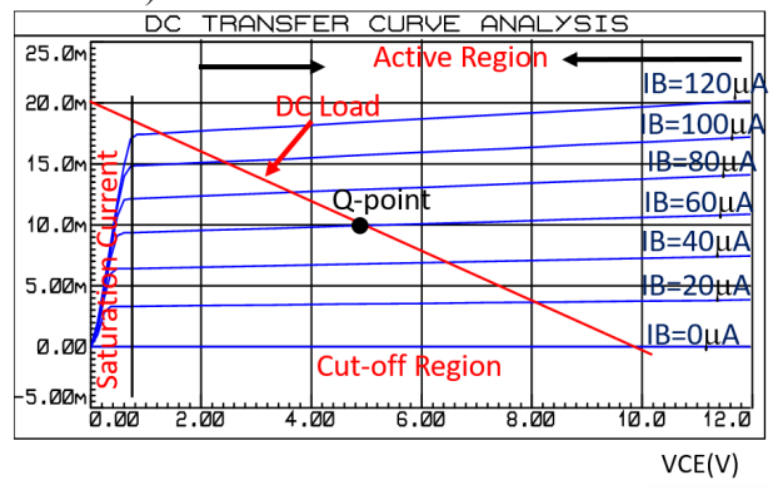

Fig. 8: Output results characterization of a) PNP configuration; b) NPN configuration using Proteus 8 software.

From these two figures, students can learn from these figures the followings:

- For PNP transistors, the curve (Fig. 8 a) is plotted between the output current $\left(\mathrm{I}_{\mathrm{C}}\right)$ and input voltage $\left(\mathrm{V}_{\mathrm{BC}}\right)$ for different input Emitter current $\left(\mathrm{I}_{\mathrm{E}}\right)$ values $(0-2.5 \mathrm{~mA})$. 
- $\quad$ For NPN transistors, the curve (Fig. 8 b) is plotted between the output current $\left(\mathrm{I}_{\mathrm{C}}\right)$ and output voltage $\left(V_{C E}\right)$ for different input Base current $\left(I_{B}\right)$ values $(0-120 \mu \mathrm{A})$.

- Both transitions output characteristics curves have three regions of interest stated as an active or operation region, cut-off region when the Tr acts as an 'OFF' switch, and saturation region when the Tr acts as an 'ON' switch.

- In the Cut-off region, both the Base Emitter junction and Collector Base junction are reverse biased $\left(\mathrm{V}_{\mathrm{CC}}=\mathrm{V}_{\mathrm{BC}}\right.$ for PNP and $\mathrm{V}_{\mathrm{CC}}=\mathrm{V}_{\mathrm{CE}}$ for NPN; because $\left.\mathrm{I}_{\mathrm{c}}=0\right)$, while in the saturation area, both the Base Emitter junction and Collector Base junctions are forward biased $\left(\mathrm{V}_{\mathrm{CC}}=\mathrm{I}_{\mathrm{C}} \mathrm{xR}\right.$ 保 because $\mathrm{V}_{\mathrm{BC}}$ and $\mathrm{V}_{\mathrm{CE}}=0$ for PNP and NPN respectively).

- Whereas in the active region, the Base Emitter junction is connected in the forward-biased whereas the collector-base junction is connected in the reverse-biased $\left(V_{C C}=I_{C} \times R_{L}+V_{B C}\right.$ for PNP and $V_{C C}=I_{C} x R_{L}+V_{C E}$ for NPN) [14].

More information about the open/closed transistor switching characteristics is explained in table 3.

Student can build up their practice transistor switching as shown in Fig. 9. In this figure at the cut-off region, the transistor is off because the input base current $\left(\mathrm{I}_{\mathrm{B}}\right)$ and output collector current $\left(\mathrm{I}_{\mathrm{C}}\right)$ are zero. This means that the maximum voltage across the collector and the emitter are $\mathrm{V}_{\mathrm{CC}}$ as the current is not passing through the device due to the large depletion layer. Therefore, the transistor is switched "fully-off". While in Fig. 9 b) at the saturation region, the transistor is biased with the maximum value of $\mathrm{I}_{\mathrm{B}}$, which results in a maximum $\mathrm{I}_{\mathrm{C}}$. This means that the collector-emitter voltage is dropped to a minimum and hence a maximum current passing through the transistor because the depletion layer is as small as possible. Therefore, the transistor is switched "fully-on". 


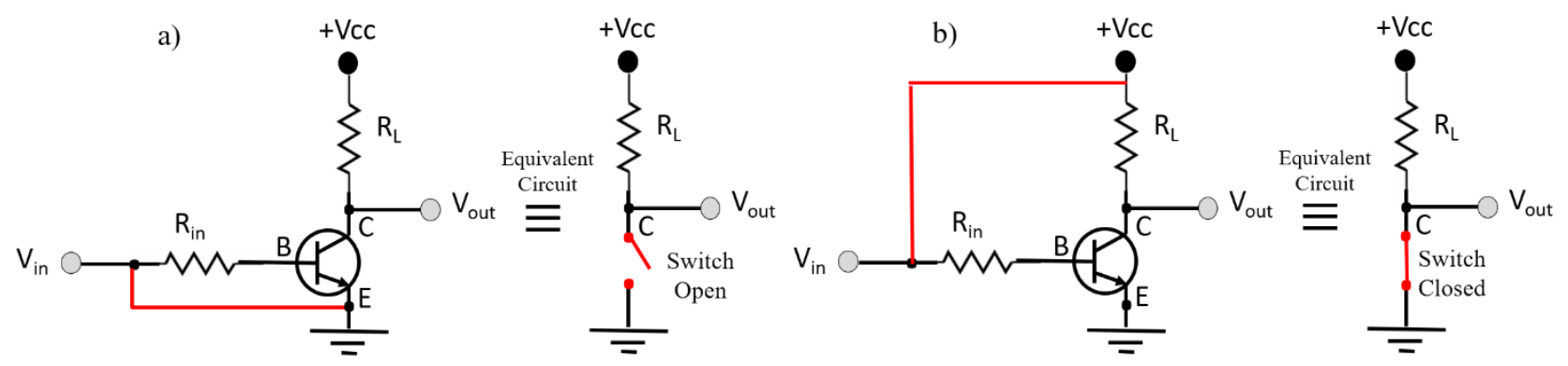

Fig. 9: a) Cut-off Characteristics; b) Saturation characteristics [14].

Table (3): The characteristics differential between open and closed switching transistors.

\begin{tabular}{|l|l|}
\hline Cut-off Characteristics (open switch) & Saturation characteristics (closed switch) \\
\hline The Base is at $0 \mathrm{~V}$ & The input and Base are connected to $\mathrm{V}_{\mathrm{CC}}$ \\
\hline Base-Emitter voltage $\mathrm{V}_{\mathrm{BE}}<0.7 \mathrm{~V}$. & Base-Emitter voltage $\mathrm{V}_{\mathrm{BE}}>0.7 \mathrm{~V}$ \\
\hline Base-Emitter junction is reverse biased & Base-Emitter junction is forward biased \\
\hline Base-Collector junction is reverse biased. & Base-Collector junction is forward biased \\
\hline $\begin{array}{l}\text { The transistor is OFF in this region (Cut-off } \\
\text { region) }\end{array}$ & The transistor is ON in this region \\
\hline The Collector current flows is zero ( $\left.\mathrm{I}_{\mathrm{C}}=0\right)$ & Max Collector current flows ( $\left.\mathrm{I}_{\mathrm{C}}=\mathrm{Vcc} / \mathrm{R}_{\mathrm{L}}\right)$ \\
\hline $\mathrm{V}_{\mathrm{OUT}}=\mathrm{V}_{\mathrm{CE}}=\mathrm{V}_{\mathrm{CC}}=$ "1" & $\mathrm{V}_{\mathrm{OUT}}=\mathrm{V}_{\mathrm{CE}}=$ "0" (ideal saturation) \\
\hline Transistor operates as an "open switch" & Transistor operates as a "closed switch" \\
\hline
\end{tabular}

VI. Integrated circuit (IC): ICs are mainly taught to a higher level at the university level and also are widely used in student graduate projects and postgraduate research studies. It is a set of active components (e.g., transistors and diodes) and passive components (e.g., capacitors and resistors) with multi-terminals that fabricated as a single flat chip, or a microchip that build upon a thin substrate of semiconductor material that is normally silicon by 
using a lithography technique. The integration of large numbers of these electronic components into a very small chip results in circuits that are orders of magnitude smaller, faster, and less expensive than those constructed of discrete electronic components. Today, ICs lead to the revelation of the electronics world and developed the generation of mobile phones, computers, TVs, and other digital home devices which are now inextricable in modern societies. ICs can be either Transistor-transistor logic (TTL) [15] or metal oxide semiconductor (MOS) [16] integrated circuits. ICs can be either analog or digital circuits. Analog, or linear, circuits usually utilise only a few electronic components. Generally, analog ICs circuits are used as a signal generator as shown in Fig. 10 a) and Fig. 10 b) of the NE556 schematic symbol and the circuit diagram of the dual signal generator. By implementation and simulating the circuit in fig. 10 a), a student here can learn the followings:

- How to operate the single or dual signal generator to generate a square pulse.

- Practices the electronics components and IC connections as well as supplying the right value of the voltage to the circuit.

- Calculate the signal generated by the IC556 using the following equation. Here we use $\mathrm{R}_{1}=\mathrm{R}_{2}=\mathrm{R}_{3}=10 \mathrm{~K} \Omega$ and $\mathrm{C}=0.1 \mu \mathrm{F}$, hence generating a pulse period of $1.91 \mathrm{~ms}$ (as shown in Fig. 10 e) which corresponding to the frequency of $523 \mathrm{~Hz}$ compared to $2.08 \mathrm{~ms}$ which is corresponding to the frequency of $480 \mathrm{~Hz}$ (with a percent error of $\sim 8 \%$ ) based on the calculation from the equation below:

$$
t=0.693 \times 3 R \times C_{1}=0.693\left(3 \times 10 \times 10^{3}\right) \times 0.1 \times 10^{-6}=2.08 \mathrm{~ms} .
$$

ICs are also broadly used as an operational amplifier (op-amp) as shown in Fig. 10 c) and d) for the IC741 schematic symbol and the circuit diagram of the general audio amplifier. The op-amp is a common IC that is used for increasing the size of a signal. Inside the op-amp, there is a large 
number of transistors, several resistors, and a few capacitors. A typical op-amp has an inverting (pin 2) and non-inverting (pin 3) inputs (Fig. 10 c), two DC power supply connections (positive, pin 4 and negative, pin 4), an output terminal (pin 6), and a few other specialized connections for fine-tuning the performance. After implementing and simulating this circuit using Proteus 8 software, a student here can learn the followings:

- How to operate the op-amp by amplified and invert the input signal when it is connected to pin 2, whereas can only amplify (without inverting the input signal) when it connected to pin 3.

- Calculate the amplifier value (gain) from the simulation software output figure as shown in Fig. $10 \mathrm{f}$ ) by measure the peak to peak amplitude which is about 9.98 and compared with the theoretical calculation by dividing $R_{2} / R_{1}\left(R_{2}=100 \mathrm{~K} \Omega\right.$ and $\left.R_{1}=10 \mathrm{~K} \Omega\right)$ which is equal to 10 (with a percent error of $\sim 0.2 \%$ from simulation result), this means the output electrical signal is 10 times higher than the input signal which is $1 \mathrm{~V}$.

Moreover, analog ICs circuits can be used as environmental sensors for collecting data and monitoring the environment such as the temperature and pressure sensors. 
a)

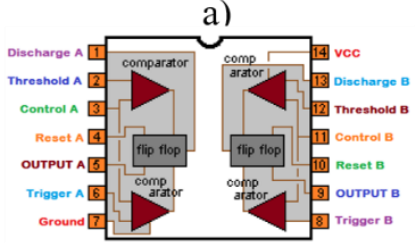

b)

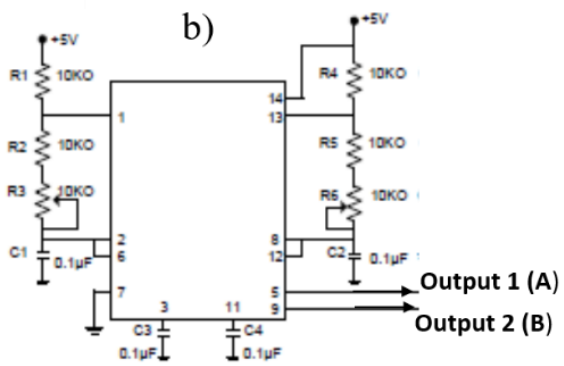

e)

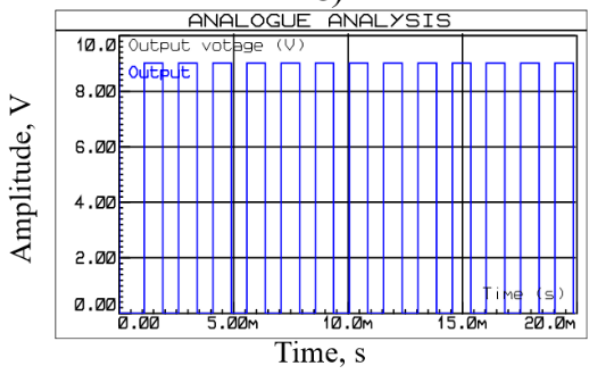

c)

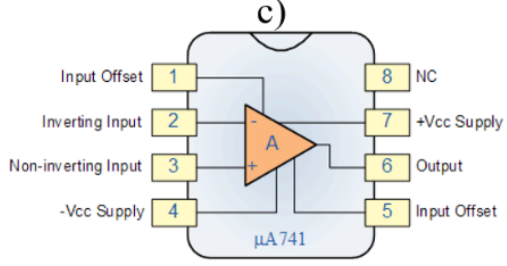

d)

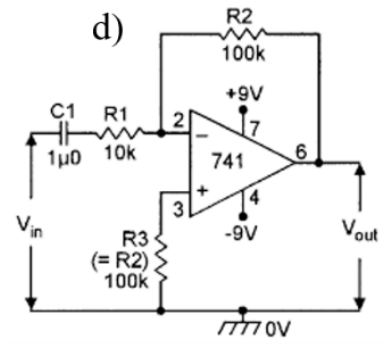

f)

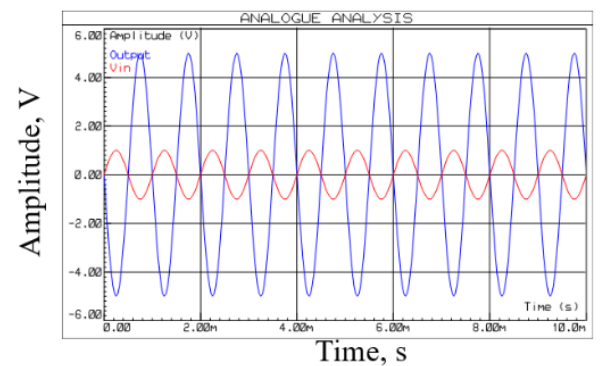

Fig. 10: a) schematic symbol, b) the circuit diagram of the NE556 dual signal generator; c) schematic symbol, d) the circuit diagram of an op-amp based on IC741, e) the generated signal from IC556 using Proteus 8 software, and f) output amplified signal from Proteus 8 software.

On the other hand, a digital circuit is designed to work with specific voltages values known as a binary circuit. It was designed with quantities of 1 and 0 representing on and off or like true and false respectively in the form of logic gates as shown in Fig 11 which shows the schematic symbol that simulates using Proteus software, truth table, and ICs circuit of the different logic gate. Different gates have been simulated in Fig. 11 which explains the output from each gate at the different input levels. In general, these gates are constructed using electronic switches such as transistors, diodes. It is used in microcontrollers and microprocessors for embedded system applications, and electronic project circuits. Therefore, these basic elements called gates are combined in the design of ICs and represent the backbone of digital computers, TVs, and modern 
electronic devices $[17,18]$. The logic gates, in general, have three terminals, two for inputs and one for output (except the NOT gate that has only one input and one output) are categorized into seven: NOT, AND, OR, NOR, XOR, NAND, and XNOR. These logic gates, their symbols, and truth tables are illustrated below.
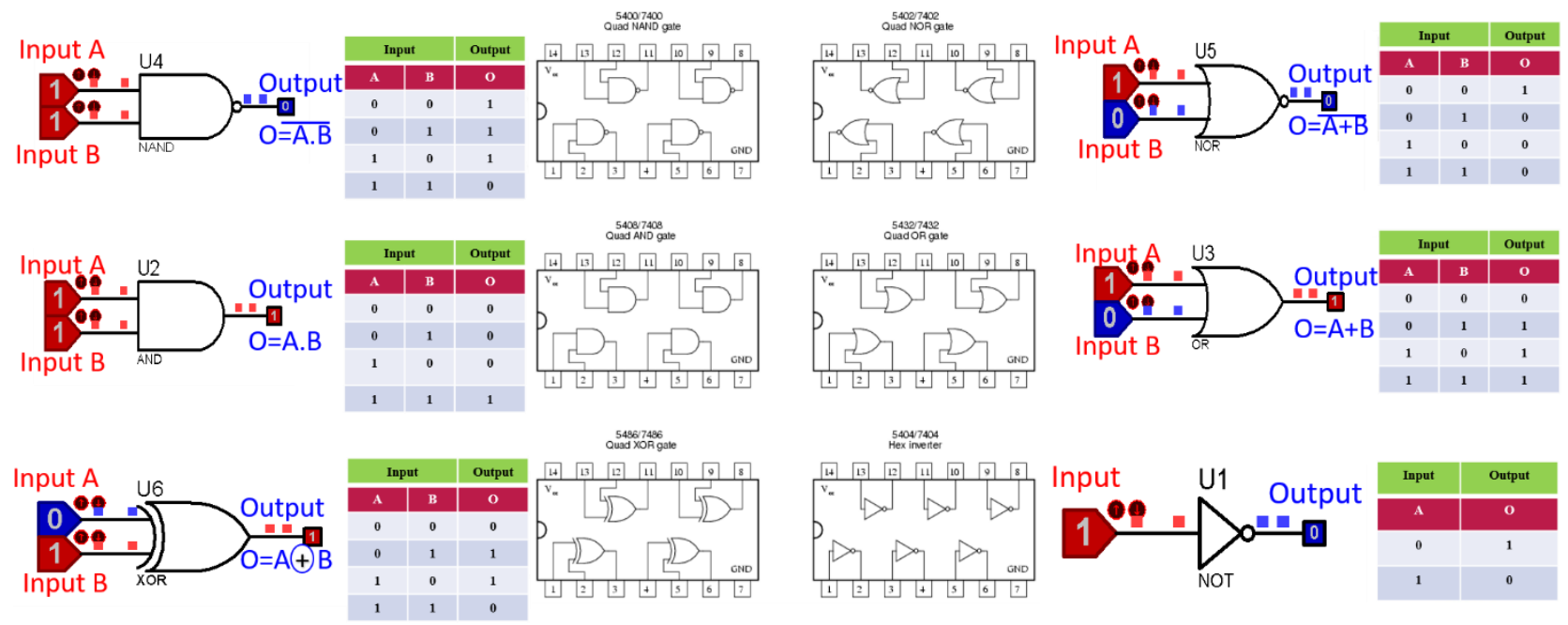

Fig. 11: Schematic symbol, truth table, and ICs circuit [19] of the digital logic gate.

NOT Gate: This only the gat that has one input and one output. The output of this gate is the reverse of the input; therefore, it operates as an inverter of the input. For example, in the case of the input signal is 1 (true), the output from this gat is 0 (false) and vice versa.

AND Gate: This gate has two inputs and one output. The output of this gate is 1 (true) only when all the inputs are 1 . Whereas, the output is 0 when one or both inputs are 0 (false).

OR Gate: The output of the OR gate is 1 when one or both inputs are 1 whereas the output is 0 when both inputs are 0 .

NAND Gate: This gate is the combination of the AND and NOT gates, employing AND gate followed by NOT gate. If the two inputs of this gate are 1 , then the output of the gate will be 0 , where the output is 1 when one of the inputs is 0 . So, it is exactly the reverse of the AND gate. 
NOR Gate: The NOR gate carries out the principle operation of the OR gate followed by the NOT gate (combining the OR and NOT gates). When any one of the inputs of this gate is 1 , then the output will be 0 . It is exactly the reverse of the OR gate.

Exclusive-OR Gate (XOR): The XOR gate performs based on the operation of the OR gate. If any of the two inputs is 1 , then the output from this gate is 1 . Whereas the output is 0 when both inputs are either 1 or 0.

Exclusive-NOR Gate (XNOR): XNOR gate performs based on the operation of NOR gate. The output from this gate is 1 when both inputs are either 1 or 0 . Whereas the output is 0 when the two inputs are different.

VII. Microprocessors circuits are the most complex integrated circuits that contained billions of transistors configured into thousands of individual digital circuits, each one performing a specific logic function [20]. Teaching the principle of the microprocessors to undergraduate and postgraduate students is important to expand their knowledge about using this device in a different practice setup and devices. So, the students can widen their knowledge about the concepts and architecture of this device and also learn how to drive and communicate with the electronic device through complete assembly language programs (software). The microprocessor is built completely from the logic gates in Fig. 11 which are synchronized to each other. So, the microprocessors model includes many components such as registers, memory the central processing unit (CPU) of a computer, and some other piece of electronic equipment. Figure 12 a and $\mathrm{b}$ shows the picture and schematic symbol of the 80C51 microprocessors which have 4 parallel input ports from $\mathrm{P}_{0}$ to $\mathrm{P}_{3}$, and each port uses 8 pins.

This device can be programmed to work as time-division multiplexing for communicating many data users within nano-second (part of billions) time [21]. Normally, the 8051 microcontrollers 
are widely used in touch screens of media players, gaming devices, and cell phones; medical devices such as handy heart rate and blood pressure; in hybrid motor vehicles automobiles; and in energy management of calculating energy consumption applications.

a)

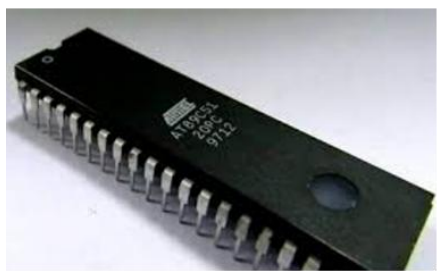

b)

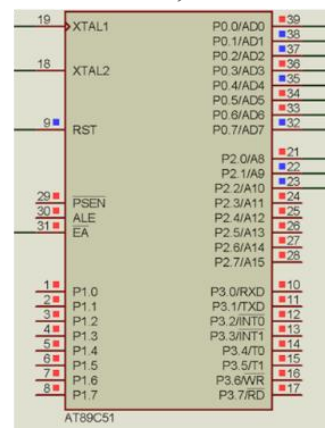

Fig. 12: a) and b) showing the picture and schematic symbol of the $80 \mathrm{C5} 1$ microprocessors.

VIII. Arduino: is a built-in open-source electronics platform consisting of easy-to-use hardware equipment and software programs. This item is designed and manufactured as single-board microcontrollers (microcontroller kits) for supporting a diverse group of professional students in building digital devices for different applications. It is also aimed at people who want to use programmable-electronic hardware without needing to investigate deeply into how a microcontroller operates. This platform is increasingly used in mechanical engineering programs. Arduino boards are designed based on the use of a set of microprocessors and controllers as shown in Fig. $13 \mathrm{a}$ and $\mathrm{b}$ for ATmega microcontroller 328P and 2560 respectively. Both are equipped with a variety of analog and digital input/output (I/O) pins as shown in Fig. 13c for the ATmega microcontroller 328P board layout that may be interfaced to different expansion boards and other circuits, more details about the Aurdino board, block and circuit diagrams are available in $[22,23]$ as well as in the data sheets and manual user. The Arduino includes all of the software and hardware needed to be powered and programmed through Universal Serial Bus (USB), and all of its inputs and outputs are brought out on 
connectors running on two edges. On the Arduino Uno, digital I/O pins are given the numbers 0 to 13 (see figure 13), and within the Arduino software, any reference to 5, for example, will be a reference to digital pin 5 . The boards have a feature called serial communications interfaces that includes micro USB for communication through computer and loading software programs to drive the device based on the application. The board has also designed supplied with 5V DC through the USB connection or also could be through the Vin pin. Microcontrollers can be programmed using $\mathrm{C}++$ programming languages. The Arduino project, therefore, offers a control line tool as an integrated development environment device. It has several facilities for communicating with another electronic device such as with another Arduino board, computer, sensors, microcontrollers, the communication between Arduino and other devices can be done via the TTL $(5 \mathrm{~V})$ serial communication or through USB serial communication that appears as a virtual com port to software on the computer. The transmitter (Tx) and receiver (RX) LEDs on the board will flash when data is being transmitted via the USB connection to the computer.

The Arduino UNO is designed for students, programmers, designers, beginners, and hobbyists. It is relatively inexpensive, simple, open-source, and extensible hardware, open-source and extensible software, as well as that, can run on many operating systems (Windows, Macintosh OSX, and Linux). Therefore, this board became an ideal, entry-level board for student projects who are new to developing on the Arduino platform as well as for the postgraduate and researchers for rapid prototyping. In addition, students can expand their knowledge with Arduino by practices the construction of low-cost scientific electronic circuits and devices, to explain the principles of electronics and physics, or to begin with programming and robotics. For instance, it can demonstrate the basics of sensors and actuators as well as a perfect introduction to electronics and coding. The Arduino board started recently adapted with new electronic demands and 
challenges, showing its offer from simple 8-bit boards to products for Internet-of-things (IoT) applications, wearable, 3D printing, and embedded environments [24-27]. Another example, designers and architects are able now to build interactive musical instruments based on the Arduino board such as laser harp. Moreover, the Arduino project features are wireless control, automation, motors control, robots arm and cars, Radar, range measurer, computer numerically controlled (CNC) foam cutting machine, RC airplane, LEDs matrix scrolling text, and thousands of different projects and applications. For instance, Fig. 14 a and b show LED matrix scrolling text Arduino and the robot car wireless control projects respectively. Further to the wide applications of such robotic cars in the environment based on IoT, it has also become hobbies of middle and high school students, who design and operate them in clubs and scientific societies.

For the novel COVID19 pandemic battle, the Arduino board helped the society in this fighting through developed new devices such as automatic sanitization machine, contactless temperature screening, and cough detection system [28, 29] to distinguish between the noise and cough sound, hence detect the Coronavirus suspect by machine learning techniques.

a)

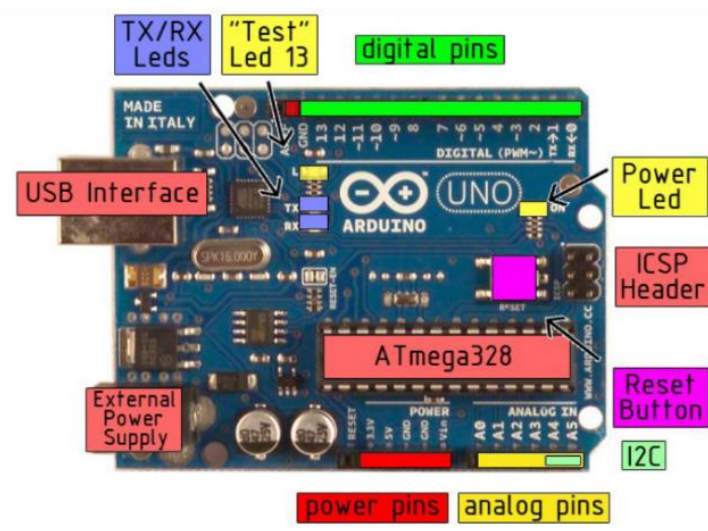

b)

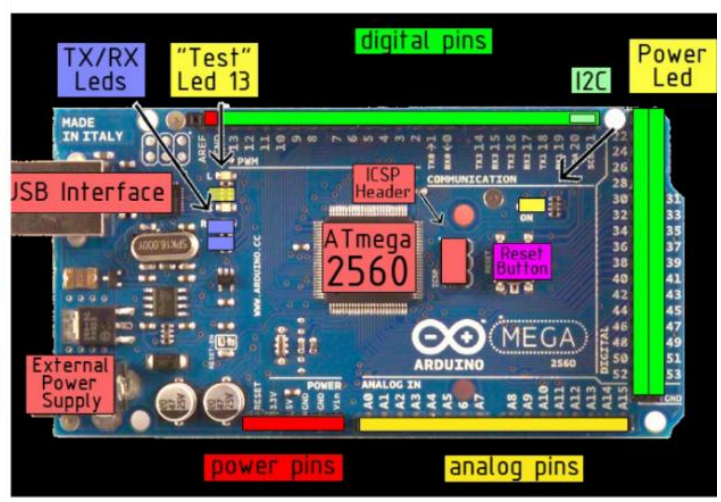




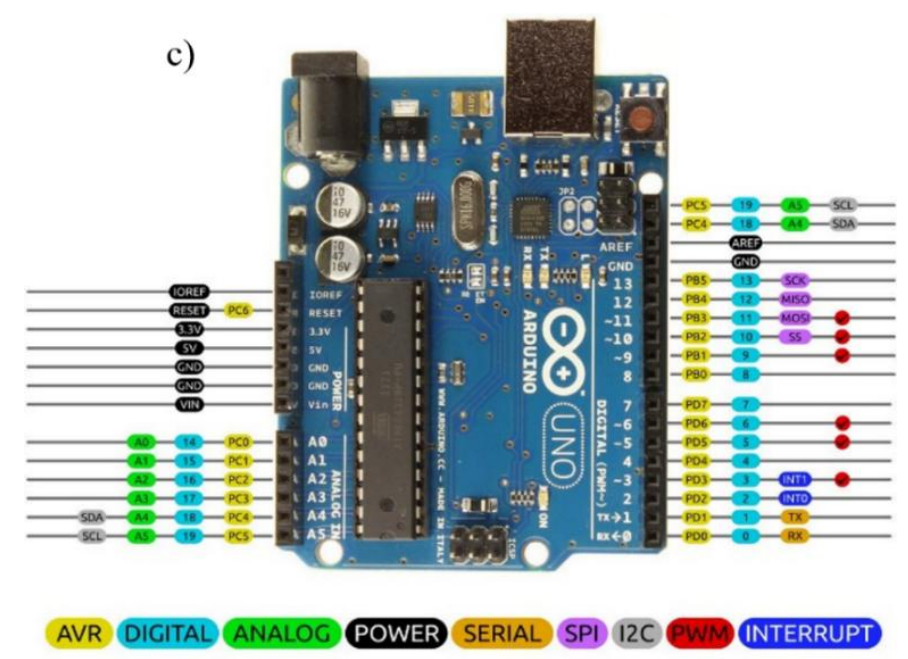

Fig.13: Arduino board based on ATmega microcontroller, a) 328P, b) 2560 and c) ATmega microcontroller 328P board layout [22,23].

a)

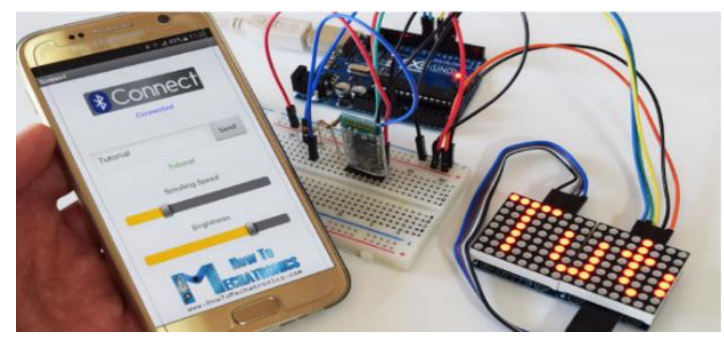

b)

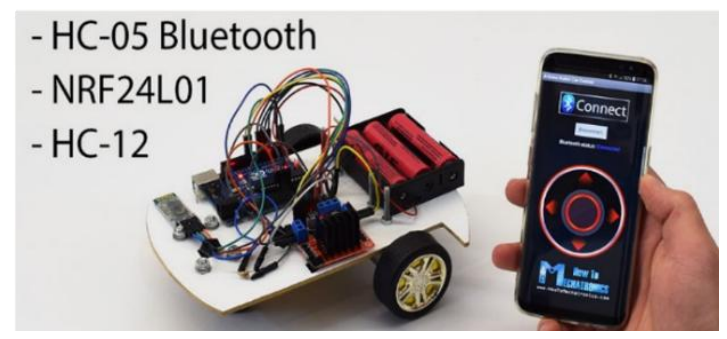

Fig. 14: (a): LED Matrices control system through a smartphone, (b) Robot car wireless with three different control methods HC-05 Bluetooth module, the NRF24L01 transceiver module, and the HC-12 long-range wireless module [24-27].

IX. Raspberry $\mathbf{P i}$ : is a compact and small single computer board that offers endless opportunities, particularly for promoting teaching the concepts of the basic computer for undergraduate and postgraduate students. It is a high speed, connectivity, and simply connected through the IoT into your workplace (laptop, mouse, keyboard, power supply), robotic and even TV, further to make complex computer-controlled systems, and, hence providing various functionalities. Because of the low cost and portability of this device, it is now used extensively by students in their final year projects, research projects, and many other applications such as robotic, communication, and weather monitoring. Moreover, there are also add-on boards 
available to enable other uses, such as camera, liquid-crystal display (LCD) screen modules, media players, education to gaming, IoT monitoring integration working conditions, work productivity, and other many ranges of applications. Raspberry has also developed through the years and all models at the moment contains featured of Broadcom system on a chip with an integrated Random-Access Memory (RAM)-compatible central processing unit (CPU) and onchip graphics processing unit (GPU) as shown in Fig. 15. It has up to $1.5 \mathrm{GHz}$ process speed and 2 GB RAM [30]. The boards also have 1-5 USB ports, HDMI for video output, and tip-ring audio output as well as Wi-Fi and Bluetooth. Therefore, $\mathrm{Pi}$ is an excellent candidate for wired and wireless data, audio and video communication systems as well as for environmental sensing applications.

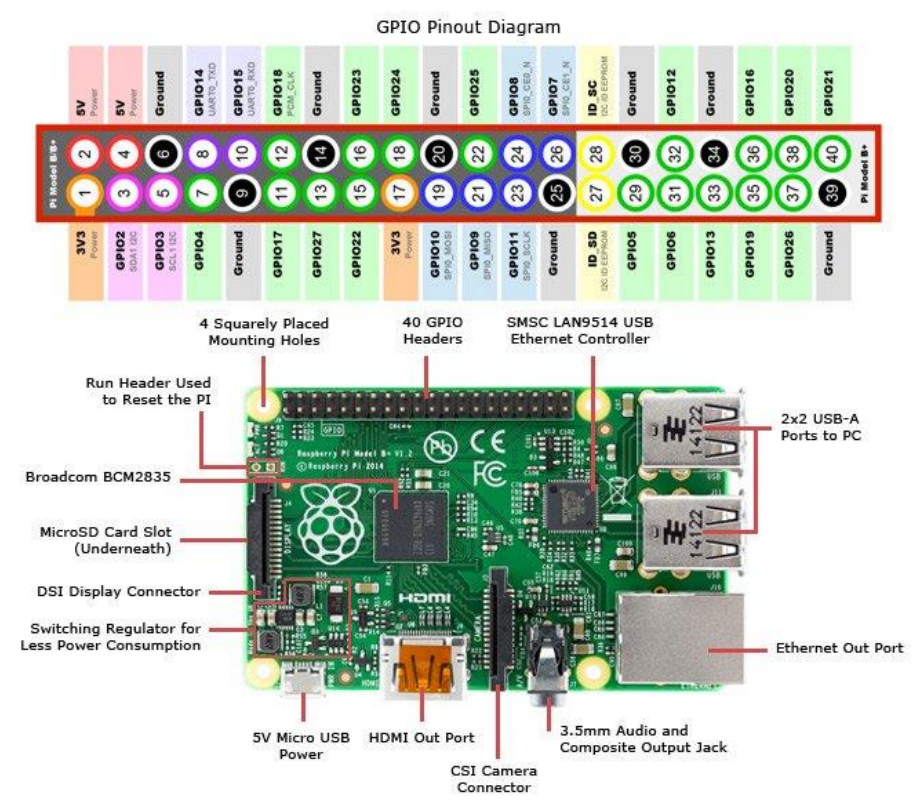

Fig. 15: Raspberry board layout [30]. 


\section{Conclusions:}

Within the hard situation similar to the COVID-19, students believe that they do not have the capacity to carry out electronic devices and projects themselves and that their studies are not sufficient to receive such skills, but it is believed that what has been reviewed above will help that students to have the capabilities and skills to localize and work independently with such techniques. Proteus 8 simulation software offered a solution for online laboratory learning in the absence of the physical laboratory due to the national lockdown and social distance. Proteus simulation results are very good in agreement with the theoretical calculations for all the electronic circuits were simulated using this software. It is more important for the teacher in the online class and future normal classroom to conduct an existed and new experiment with the students and request the implementation of projects with open specifications that leave the freedom for students to choose what they want to do. This will expand the practical design and implantation skill, hence, expand the knowledge of the students which leads them to build up their projects that can be connected with IoT for future applications. Furthermore, this study will give an opportunity for hard-working students interested in studying and learning to innovate, research and gain experience while at the same time a great challenge for students who are not properly exploited. The general goal of students will always be the attention to study, education, and the desire for a distinctive and good achievement. 


\section{List of Abbreviations.}

\begin{tabular}{|l|l|l|}
\hline 1 & AC & Alternating current \\
\hline 2 & CNC & computer numerically controlled \\
\hline 3 & CPU & central processing unit \\
\hline 4 & DC & Direct current \\
\hline 5 & FET & field-effect transistor \\
\hline 6 & GPU & graphics processing unit \\
\hline 7 & IC & Integrated circuit \\
\hline 8 & I/O & analogue input/output \\
\hline 9 & IoT & Internet-of-things \\
\hline 10 & LCD & liquid-crystal display \\
\hline 11 & LED & light-emitting diode \\
\hline 12 & MOS & Metal oxide semiconductor \\
\hline 13 & op-amp & operational amplifier \\
\hline 14 & RAM & Random-Access Memory \\
\hline 15 & TTL & Transistor-transistor logic \\
\hline
\end{tabular}




\section{References}

1. T. R. Crompton, "Battery Reference Book," (third ed.). Newnes. ISBN 9780080499956 , 2000.

2. L. Pauling, " General Chemistry,". New York: Dover Publications, Inc. p. 539. ISBN 9780486656229, 1988.

3. K. Schmidt-Rohr, "How Batteries Store and Release Energy: Explaining Basic Electrochemistry". Journal of Chemical Education. 95 (10), 1801-1810, 2018.

4. A. M. Robert and E. S. Bishop, Elements of Electricity," Chicago, American Technical Society, 1917.

5. J. D. Jackson, "Surface charges on circuit wires and resistors play three roles," American Journal of Physics, 64, 855-870, 1996.

6. R. S. Walter and R. H. Suk, "Trick of the Trade: A Series-Parallel Demonstration," TPT 33, 590, 1995.

7. F. Y. Wu, "Theory of resistor networks: The two-point resistance". Journal of Physics A: Mathematical and General. 37 (26): 6653-6673, 2004.

8. https://www.electronics-tutorials.ws/blog/i-v-characteristic-curves.html

9. F. T. Ulaby, "Fundamentals of Applied Electromagnetics," Upper Saddle River, NJ: Prentice Hall, Page 168. ISBN 9780130115546, 1999.

10. D. Janzen, "Introduction to Electricity, Magnetism, and Circuits," a Creative Commons Attribution 4.0 International License, University of Saskatchewan, Distance Education Unit, Canada, November 28, 2018.

11. L. J. Giacoletto, "Electronics Designers," Handbook. New York: McGraw-Hill, 1977.

12. D. L. Pulfrey, "Understanding Modern Transistors and Diodes," Cambridge University Press. ISBN 9781139484671, 2010.

13. https://www.electronics-tutorials.ws/amplifier/amp_2.html

14. PNP Transistor - Working Principle, Characteristics \& Applications, https://electricalfundablog.com/

15. D. Lancaster, "TTL Cookbook", Howard W. Sams and Co., Indianapolis, ISBN 0672210355, 1975.

16. Y. Kuo, "Thin Film Transistor Technology-Past, Present, and Future," The Electrochemical Society Interface. 22 (1): 55-61, 2013.

17. A. Maini, Digital Electronics: Principles, Devices and Applications," John Wiley \& Sons. p. 168. ISBN 9780470032145, 2007.

18. R. L. Morris and J.R. Miller, "Designing with TTL Integrated Circuits," Texas Instruments Electronics Series, McGraw-Hill, New York, 1971.

19. https://www.technocrazed.com/3-11-dip-gate-packaging.

20. A. Osborne, “An Introduction to Microcomputers," Volume 1: Basic Concepts (2nd ed.). Berkeley, California: Osborne-McGraw Hill, ISBN 9780931988349, 1980.

21. H. A. Mohammed, H. J. Kbashi, A. N. Almaamory, "Design and Implementation of a Network Based on Wavelength Division Multiplexing (WDM), Journal of Kerbala university 9 (1), 2011.

22. D. Kushner, "The Making of Arduino," IEEE Spectrum, 26 Oct. 2011. 
23. J. Lahart, "Taking an Open-Source Approach to Hardware," The Wall Street Journal, Nov. 2009.

24. https://howtomechatronics.com/arduino-projects/

25. R. Bardaji, A. M. Sánchez, C. Simon, M. Wernand, J. Piera, "Estimating the Underwater Diffuse Attenuation Coefficient with a Low-Cost Instrument: The KdUINO DIY Buoy," Sensors. 16 (3): 373. 2016.

26. P. Beddows, E. Mallon, "Cave Pearl Data Logger: A Flexible Arduino-Based Logging Platform for Long-Term Monitoring in Harsh Environments," Sensors. 18 (2): 530, 2018.

27. A. S. Ali, Z. Zanzinger, D. Debose, B. Stephens, "Open Source Building Science Sensors (OSBSS): A low-cost Arduino-based platform for long-term indoor environmental data collection," Building and Environment. 100: 114-126, 2016.

28. https://create.arduino.cc/projecthub/edge-impulse/cough-detection-with-tinyml-on-arduino$417 \mathrm{f} 37$.

29. M. Thangamani, M. Ganthimathi, S.R. Sridhar, M. Akila, R. Keerthana, P.S. Ramesh, "Detecting coronavirus contact using internet of things," International Journal of Pervasive Computing and Communications, ISSN: 1742-7371, Publication date: 22 July 2020.

30.G. Halfacree, Gareth. "Raspberry Pi 4 now comes with 2GB RAM Minimum," The .30 MagPi (91). Raspberry Pi Press, , 2020. 